\title{
Alpha-particle clustering in excited expanding self-conjugate nuclei
}

B. Borderie ${ }^{1}$, Ad. R. Raduta ${ }^{1,2}$, G. Ademard ${ }^{1}$, M. F. Rivet ${ }^{1 \dagger}$,

E. De Filippo ${ }^{3}$, E. Geraci ${ }^{3}$, N. Le Neindre ${ }^{1,4}$, G. Cardella $^{3}$, G. Lanzalone ${ }^{5}$, I. Lombardo ${ }^{5}$, O. Lopez $^{4}$, C. Maiolino ${ }^{5}$,

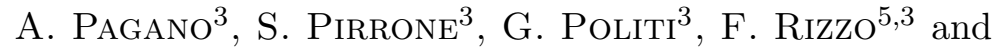
P. Russotto $0^{5,3}$

${ }^{1}$ Institut de Physique Nucléaire, CNRS/IN2P3, Université Paris-Sud 11, 91406 Orsay, France

2 National Institute for Physics and Nuclear Engineering, Bucharest-Măgurele, Romania

3 INFN, Sezione di Catania and Dipartimento di Fisica e Astronomia, Università di Catania, Italy

${ }^{4}$ LPC, CNRS/IN2P3, Ensicaen, Université de Caen, Caen, France

${ }^{5}$ INFN, Laboratori Nazionali del Sud, Catania, Italy

\begin{abstract}
The fragmentation of quasi-projectiles from the nuclear reaction ${ }^{40} \mathrm{Ca}+{ }^{12} \mathrm{C}$ at $25 \mathrm{MeV} /$ nucleon was used to produce $\alpha$-emission sources. From a careful selection of these sources provided by a complete detection and from comparisons with models of sequential and simultaneous decays, strong indications in favour of $\alpha$-particle clustering in excited ${ }^{16} \mathrm{O},{ }^{20} \mathrm{Ne}$ and ${ }^{24} \mathrm{Mg}$ are reported.
\end{abstract}

\section{Introduction}

Clustering is a generic phenomenon which can appear in homogeneous matter when density decreases; the formation of galaxies as well as the disinte-

${ }^{\dagger}$ Deceased 
gration of hot dilute heavy nuclei into lighter nuclei are extreme examples occuring in nature. As far as nuclear physics is concerned, the nucleus viewed as a collection of $\alpha$-particles was very early discussed and in the last forty years both theoretical and experimental efforts were devoted to clustering phenomena in nuclei. Very recently the formation of $\alpha$-particle clustering from excited expanding self-conjugate nuclei was revealed in two different constrained self consistent mean field calculations $[1,2]$. The aim of the present work was to obtain, from the experimental side, some information on $\alpha$-particle clustering from excited and consequently expanding alpha-conjugate nuclei. The chosen experimental strategy was to use the reaction ${ }^{40} \mathrm{Ca}+{ }^{12} \mathrm{C}$ at an incident energy $(25 \mathrm{MeV}$ per nucleon) sufficient to possibly produce some hot expanding reaction products, associated with a high granularity-high solid angle particle array (to precisely reconstruct directions of velocity vectors). Then, by selecting the appropriate reaction mechanism and specific events the required information was derived.

\section{Experimental details and event selection}

The experiment was performed at INFN, Laboratori Nazionali del Sud in Catania, Italy. The beam impinging on a thin carbon target $\left(320 \mu \mathrm{g} / \mathrm{cm}^{2}\right)$ was delivered by the Superconducting Cyclotron and the charged reaction products were detected by the CHIMERA $4 \pi$ multi-detector [3]. The beam intensity was kept around $10^{7}$ ions/s to avoid pile-up events. CHIMERA consists of 1192 telescopes ( $\Delta \mathrm{E}$ silicon detectors 200-300 $\mu \mathrm{m}$ thick and $\mathrm{CsI}(\mathrm{Tl})$ stopping detectors) mounted on 35 rings covering $94 \%$ of the solid angle, with very high granularity at forward angles. Details on $\mathrm{A}$ and $\mathrm{Z}$ identifications and on the quality of energy calibrations can be found in refs. [3-5]. Energy resolution was better than 1\% for silicon detectors and varies between 1.0 and $2.5 \%$ for alpha particles stopped in $\mathrm{CsI}(\mathrm{Tl})$ crystals.

As a first step in our event selection procedure, we want to exclude from the data sample poorly-measured events. Without making any hypothesis about the physics of the studied reaction one can measure the total detected charge $Z_{t o t}$ (neutrons are not measured). In relation with their crosssections and with the geometrical efficiency of CHIMERA, the well detected reaction mechanisms correspond to projectile fragmentation $(\mathrm{PF})[6-8]$ with $Z_{t o t}=19-20$ (target fragmentation not detected) and to incomplete/complete fusion with $Z_{t o t}=21-26$. At this stage we can have a first indication on the multiplicity of $\alpha$-particles, $M_{\alpha}$, emitted per event for well detected events ( $Z_{t o t} \geq 19$ - see fig.1). $M_{\alpha}$ extends up to thirteen, which means a deexcita- 


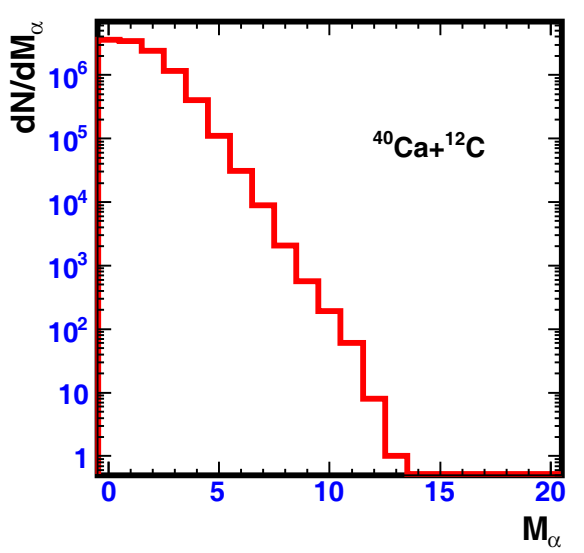

Figure 1: Distribution of $\alpha$-particle multiplicity, $M_{\alpha}$, for well detected events $\left(Z_{t o t} \geq 19\right)$

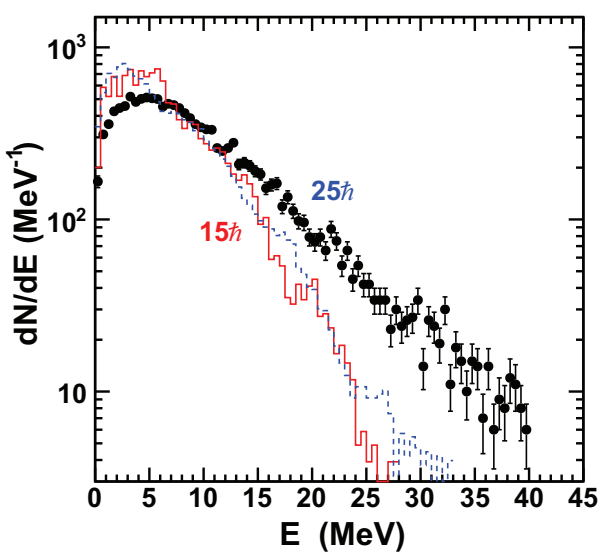

Figure 2: Sequential decay of excited $\mathrm{Ca}$ projectiles: energy spectra (in the $N \alpha=5$ system reference frame) of evaporated $\alpha$ particles associated to a ${ }^{20} \mathrm{Ne}$ evaporation residue. Full points are experimental data and histograms are results of GEMINI simulations (see text)

tion of the total system into $\alpha$-particles only. Moreover a reasonable number of events exhibit $M_{\alpha}$ values up to about 6 .

The goal is now to tentatively isolate, in events, reaction products emitting $\alpha$-particles only. To do this, knowing that at such incident energy ${ }^{40} \mathrm{Ca}$ as ${ }^{20} N e \mathrm{PF}$ is dominated by alpha-conjugate products [6], we restrict our selection to completely detected PF events $\left(Z_{t o t}=20\right)$ composed of one projectile fragment and of four to six $\alpha$-particles. Charge conservation imposes $Z_{\text {frag }}=20-2 M_{\alpha}$.

After this double selection, the question is: from which emission source are the $\alpha$-particles emitted? Several possibilities have to be considered and complementary selections must be done before restricting our study to alphasources emitting exclusively the $M_{\alpha}$ observed (called $N \alpha$ sources in what follows). Possibilities associated to selected PF events are the following:

I) considering the incident energy of the reaction and the strong forward focusing of reaction products, it is important to identify the possible presence of preequilibrium (PE) $\alpha$-particles in our selected PF events. With the hypothesis that all the $\alpha$-particles are emitted from their centre-of-mass reference frame, we noted a thermal distribution with the presence of a high energy tail starting at $40 \mathrm{MeV}$, which signs PE emission. Events in 
which such PE emission was possibly present were suppressed to prevent errors on alpha emitter properties; an upper energy limit was imposed to the $\alpha$-particle energy, with a value of $40 \mathrm{MeV}$ irrespective of $M_{\alpha}$.

II) $\alpha$-particles can be emitted from deexcitation of PF events via unbound states of ${ }^{12} \mathrm{C},{ }^{16} \mathrm{O},{ }^{20} \mathrm{Ne}$ and not directly from excited expanding $N \alpha$ sources. Multi-particle correlation functions $[5,9]$ were used to identify unbound states $100 \% \alpha$-particle emitters and suppress a small percentage of events (1.6-3.6\%).

III) it must be verified that the fragments associated with $M_{\alpha}$ are not the evaporation residues of excited $C a$ projectiles emitting only $\alpha$-particles.

As far as the two first items are concerned the effect was to suppress from $8.5\left(M_{\alpha}=4\right)$ to $12.8 \%\left(M_{\alpha}=6\right)$ of previously selected events. The last item will be discussed in the following section.

To conclude on this part, one can also indicate that if excited $N \alpha$ sources have been formed their excitation energy thresholds for total deexcitation into $\alpha$-particles vary from 20 to $50 \mathrm{MeV}$ when $N \alpha$ moves from 4 to 6 . Their mean excitation energy per nucleon is rather constant around 3.3-3.5 MeV which indicates that average lowest densities around 0.7 the normal density may have been reached due to thermal pressure $[10,11]$.

\section{$3 \quad$ Evidence for $\alpha$-particle clustering}

Before discussing different possible deexcitations of $\mathrm{Ca}$ projectiles and $\mathrm{N \alpha}$ sources, information on the selected reaction mechanism is needed. Major features of PF events are reproduced by a model of stochastic transfers [8]. For primary events with $Z_{t o t}=20$ excitation energy extends up to about 200 $\mathrm{MeV}$, which allows the large excitation energy domain (20-150 MeV) measured for $N \alpha$ sources when associated to a single fragment; angular momenta extend up to $24 \hbar$, which gives an upper spin limit for $C a$ projectiles or $N \alpha$ sources.

Are $\alpha$-particles emitted sequentially or simultaneously? To answer the question $\alpha$-energy spectra are compared to simulations. For excited $\mathrm{Ca}$ projectiles and $N \alpha$ sources, experimental velocity and excitation energy distributions and distributions for spins are used as inputs. Then, results of simulations are filtered by the multi-detector replica. Simulated spectra are normalized to the area of experimental spectra.

For sequential emission the GEMINI++ code [12] was used. Before discussing $N \alpha$ sources, as said before, we must consider the possible evaporation from $\mathrm{Ca}$ projectiles. Excitation energy for projectiles is deduced from 

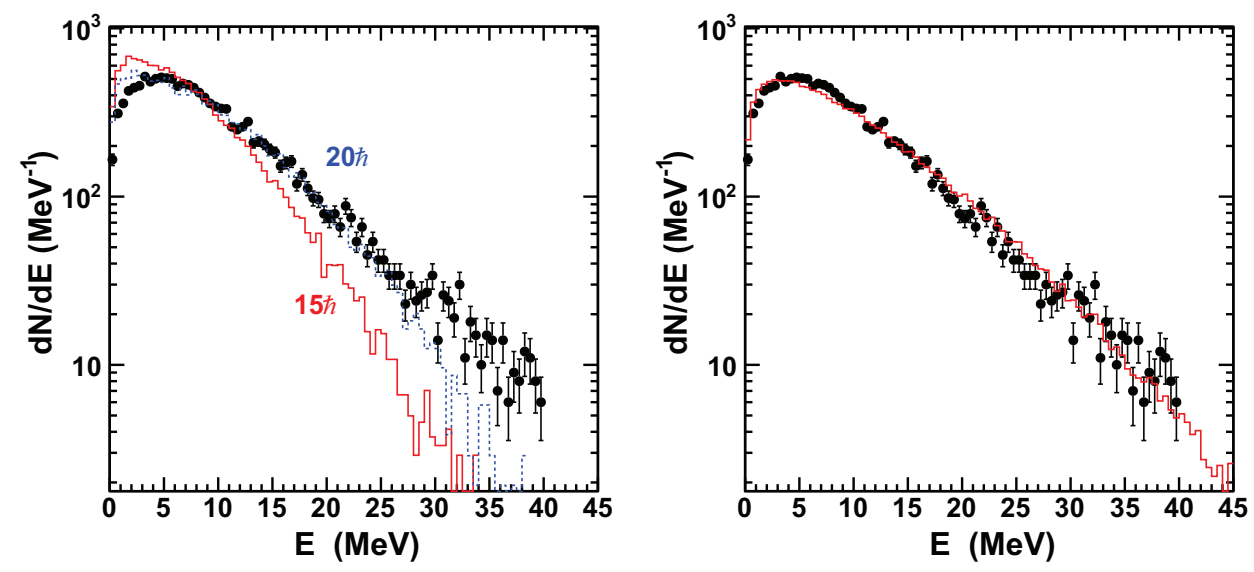

Figure 3: Decay of excited ${ }^{20} \mathrm{Ne}$ Figure 4: same as fig. 3 but histogram $(N \alpha=5): \alpha$-particle energy spectra; full corresponds to a simulation of a simultapoints are experimental data and his- neous decay (see text). tograms correspond to GEMINI simulations (see text).

$E^{*}=E^{*}(N \alpha)+E_{r e l}+Q . \quad E_{r e l}$ is the relative energy between the $N \alpha$ source and the associated fragment (evaporation residue). Fig. 2 displays results of simulations with reconstructed excitation energy distribution for ${ }^{40} \mathrm{Ca}$ $\left.\left(<E^{*}\right\rangle=88.2 \mathrm{MeV}\right)$ and gaussian distributions centred at 15 and $25 \hbar$ for spins (RMS $=1.5 \hbar)$ as inputs; note that no more ${ }^{20} \mathrm{Ne}$ residues are produced for spin distributions centred at values larger than $25 \hbar$. Comparison with experimental data shows a rather poor agreement indicating that such an hypothesis seems not correct. Same kind of results are observed for $N \alpha$ equal 4 and 6 .

Considering now excited $N \alpha$ sources only, histograms in fig. 3 are examples of GEMINI simulation results for $N \alpha=5$. Gaussian distributions for spins are used as inputs and the best agreement with data is obtained with $\mathrm{RMS}=1.5 \hbar$ for spin distributions. The agreement between data and simulations appears poorer and poorer when $N \alpha$ value decreases. Moreover an important disagreement between data and simulations is observed for the percentages of $N \alpha$ sources which deexcite via ${ }^{8} B e$ emission [13].

For simultaneous emission from $N \alpha$ sources, a simulation was done which mimics a situation in which $\alpha$ clusters are early formed when the source is expanding $[1,2]$ due to thermal pressure. The $N \alpha$ source is first splitted into $\alpha$ 's. Then the remaining available energy $\left(E^{*}+Q\right)$ is directly randomly shared among the $\alpha$-particles such as to conserve energy and linear momen- 
tum. Histogram in fig. 4 is the result of such a simulation, which shows a good agreement with data. Such agreement is also observed for $N \alpha$ equal 4 and 6. Similar histograms (within a few percents) were also obtained with simulations containing an intermediate freeze-out volume stage and then propagation in the Coulomb field.

\section{Conclusions}

In conclusion, the reaction ${ }^{40} \mathrm{Ca}+{ }^{12} \mathrm{C}$ at $25 \mathrm{MeV} /$ nucleon bombarding energy was used to produce and carefully select minor classes of events from which excited $N \alpha$ sources can be unambiguously identified. Their excitation energy distributions are derived with mean values around $3.4 \mathrm{MeV}$ per nucleon, which indicates that mean densities about 0.7 the normal density may have been reached. Their energetic emission properties were compared with two simulations, one involving sequential decays and a second for simultaneous decay. For excited expanding $N \alpha$ sources composed of 4,5 and 6 $\alpha$-particles, for which statistics is good enough for conclusives comparisons with simulations, evidence in favour of simultaneous emission ( $\alpha$-particle clustering) is reported.

\section{References}

[1] M. Girod and P. Schuck, Phys. Rev. Lett. 111 (2013) 132503.

[2] J. P. Ebran et al., Phys. Rev. C 89 (2014) 031303(R).

[3] A. Pagano et al., Nucl. Phys. A 734 (2004) 504.

[4] N. Le Neindre et al., Nucl. Instr. and Meth. in Phys. Res. A 490 (2002) 251.

[5] Ad. R. Raduta et al., Phys. Lett. B 705 (2011) 65.

[6] M. Morjean et al., Nucl. Phys. A 438 (1985) 547.

[7] B. Borderie et al., Ann. Phys. Fr. 15 (1990) 287.

[8] L. Tassan-Got and C. Stephan, Nucl. Phys. A 524 (1991) 121.

[9] R. J. Charity et al., Phys. Rev. C 52 (1995) 3126.

[10] W. A. Friedman, Phys. Rev. C 22 (1990) 667. 
[11] B. Borderie, in Large-Scale Collective Motion of Atomic Nuclei, edited by G. Giardina, G. Fazio and M. Lattuada (World Scientific, Singapore) 1997, pp. 1-14.

[12] R. J. Charity, Phys. Rev. C 82 (2010) 014610 and references therein.

[13] B. Borderie et al., Phys. Lett. B to be published. 